\title{
Critical exponent of Fujita-type for semilinear wave equations in Friedmann-Lemaître-Robertson-Walker spacetime
}

\author{
Marcelo Ebert ${ }^{1}$ and Jorge Marques $^{2}$ \\ ${ }^{1}$ University of São Paulo \\ ${ }^{2}$ University of Coimbra
}

December 27, 2021

\begin{abstract}
We consider the nonlinear massless wave equation belonging to some family of the Friedmann-Lemaitre-Robertson-Walker (FLRW) spacetime. We prove the global in time small data solutions for supercritical powers in the case of decelerating expansion universe.
\end{abstract}

\section{Hosted file}

Ebert_Marques_MMAS.pdf available at https://authorea.com/users/452826/articles/550797critical-exponent-of-fujita-type-for-semilinear-wave-equations-in-friedmann-lema $\%$ C $3 \%$

AEtre-robertson-walker-spacetime 